\title{
Homogenization on Multi-Materials' Elements: Application to Printed Circuit Boards and Warpage Analysis
}

\author{
Manuel Araújo ${ }^{1, a}$, J. L. Alves ${ }^{1}$, Paulo Silva ${ }^{1}$ and Pedro Delgado ${ }^{2}$ \\ ${ }^{1}$ MEMS, University of Minho, Dep. of Mechanical Engineering, Campus de Azurém, 4800-058 Guimarães, Portugal \\ ${ }^{2}$ Bosch Car Multimedia Portugal S.A., Apartado 2458, 4705-820 Braga, Portugal
}

\begin{abstract}
Multi-material domains are often found in industrial applications. Modelling them can be computationally very expensive due to meshing requirements. The finite element properties comprising different materials are hardly accurate. In this work, a new homogenization method that simplifies the computation of the homogenized Young modulus, Poisson ratio and thermal expansion coefficient is proposed, and applied to composite-like material on a printed circuit board. The results show a good properties correspondence between the homogenized domain and the real geometry simulation.
\end{abstract}

\section{Introduction}

One of the aims of the microelectronics field is the performance and reliability improvement [1]. In industry, weight reduction also plays an important role in a product's development cycle. Owed to the attempt of manufacturing lighter and thinner components the warpage phenomena became more noticeable.

PCBs (Printed Circuit Boards) comprise conductive and dielectric layers, consisting of copper and epoxy resin and an epoxy-woven glass fibres composite (mostly FR4), respectively (Figure 1). During the reflow soldering process, PCBs are exposed to temperatures ranging from $230^{\circ} \mathrm{C}$ to $250^{\circ} \mathrm{C}$ [2].

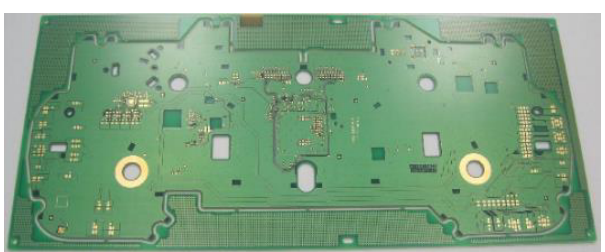

Figure 1 - Printed Circuit Board of a car cockpit.

When subjected to temperature changes, the mismatch in the thermomechanical properties of the constituent materials of the PCBs leads to the appearance of significant thermal stresses [3, 4]. Warpage is responsible for misalignments during the package assembly stage, smaller tolerances [4], imperfect soldering and consequent detachment of components [4]. In addition, it may induce cracks and the separation of the layers [5].

\subsection{Warpage problem}

The main materials of PCBs are fiberglass-reinforced (known as FR4), copper and resin. Copper and resin are

\footnotetext{
a Corresponding author: m.araujo@dem.uminho.pt
}

isotropic materials. They have the same elastic property values in every direction [6]. On the other hand, FR4 is an orthotropic material, i.e. it has three mutually perpendicular planes of elastic symmetry [7]. The key thermomechanical properties required to estimate the warpage of a PCB are the Young modulus (E), shear modulus $(\mathrm{G})$, bulk modulus $(\mathrm{K})$, Poisson ratio $(\mathrm{v})$ and the coefficient of thermal expansion $(\alpha)$. These properties fully define the materials' thermoelastic behaviour.

A PCB has always more than one conductive layer and it can contain both copper and resin. The layers have not the same geometry or copper distribution (Figure 2). For this reason there is unbalanced forces between layers.
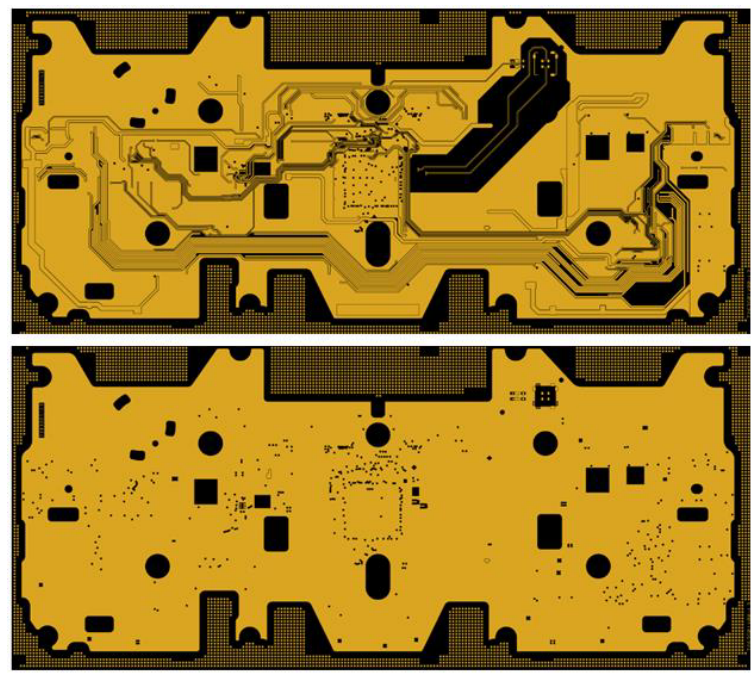

Figure 2 - Example of two layers from same PCB.

Therefore, the selection of the thermoelastic properties of the materials at the reflow soldering temperature is of great importance. 


\subsection{Warpage simulation}

For the purpose of evaluating the warpage of a PCB, the layers of the board are discretized using a finite element mesh, and then a FE analysis is performed. The fact that the conductive layers can contain both copper and resin or only one of these makes it more complex to characterize. On the other hand, the dielectric layers always have only FR4 (mainly).

The conductive layers can have copper artworks of just $80 \mu \mathrm{m}$ wide. The simulation of those geometries is very time consuming due to the complexity of the copper distribution. Furthermore, in the most cases, the computer memory space is not enough. The finite element size has to be much larger than the copper tracks wide. Thus, the calculation of homogenized thermomechanical properties is required. The methods usually applied for modelling warpage do not consider the orientation of the copper traces to calculate the PCB's homogenized properties.

Indeed, present warpage prediction techniques use volume averaging to estimate these properties [3]. Nevertheless, studies by Hutapea and Grenestedt [8] show that the copper trace orientation has a noteworthy influence on the PCBs' warpage even when the board is completely copper balanced.

\subsection{The aim of the work}

This study aims to analyse different methods for the homogenization of PCBs' conductive layers. The PCBs' constituent materials are described, as well as their most relevant thermomechanical properties.

From all the considered information, values for the different thermomechanical properties at the reflow soldering temperature (approximately $250^{\circ} \mathrm{C}$ ) were selected (Table 1). All the FEA present in this work is performed using these values. The homogenization analysis is applied to the copper layers only, and it does not take into account the FR4 layers.

First, this work takes 4 examples of simple elements of a conductive layer. The simple rule of mixtures is used and some results shown.

Then, it is presented the homogenization method, which is developed in order to become able to homogenize more properly the properties of more complex copper distribution elements. This homogenization method assumes some of the principles of the rule of mixtures.

Finally, the global results of 28 copper distribution elements are analysed. It includes some considerations about the elastic moduli, the time spent in the computation and a statistical analysis of the performance of this method.

\subsection{FEM calculation}

All models were composed by $25 \times 25 \times 1$ 8-node hexahedron finite elements. Models were created according to the original and "pixelated" Gerber file (see examples in Figure 3 and Figure 7). Its global dimensions are $1 \mathrm{~mm} \times 1 \mathrm{~mm} \times 0.04 \mathrm{~mm}$.
Table 1 - Thermomechanical properties of copper and resin.

\begin{tabular}{|l|c|c|}
\hline Property & Copper & Epoxy Resin \\
\hline$E[\mathrm{GPa}]$ & 117 & 0.070 \\
\hline$v[-]$ & 0.30 & 0.45 \\
\hline$\alpha\left[10^{-6} /{ }^{\circ} \mathrm{C}\right]$ & 21 & 197 \\
\hline
\end{tabular}

To simulate the mechanical behaviour of the model in tension, a displacement of $4 \mu \mathrm{m}$ on both in-plane directions was carried out. This value was established so the copper remained on the elastic domain. The total force was set to be null in the perpendicular directions to the imposed displacement, keeping the surfaces parallel to each other during the deformation process as a real homogeneous material would behave.

The relevant properties of the materials are the Young modulus and the Poisson ratio and the values considered for these properties are depicted in Table 1. The global Young modulus and Poisson ratio are obtained through eq. (1) and (2), respectively.

$$
\begin{gathered}
E=\frac{\sigma}{\varepsilon} \\
v=-\frac{\varepsilon_{T}}{\varepsilon_{L}}
\end{gathered}
$$

\section{Homogenization: the rule of mixtures}

Different element configurations were imported from a Gerber file (original file describing a PCB). The simpler evaluated configurations are presented in Figure 3. Those four elements, referenced as e426, e610, e614 and e625, have different distributions and area fractions of copper: $19.8 \%, 24 \%, 13.9 \%$ and $5.9 \%$.
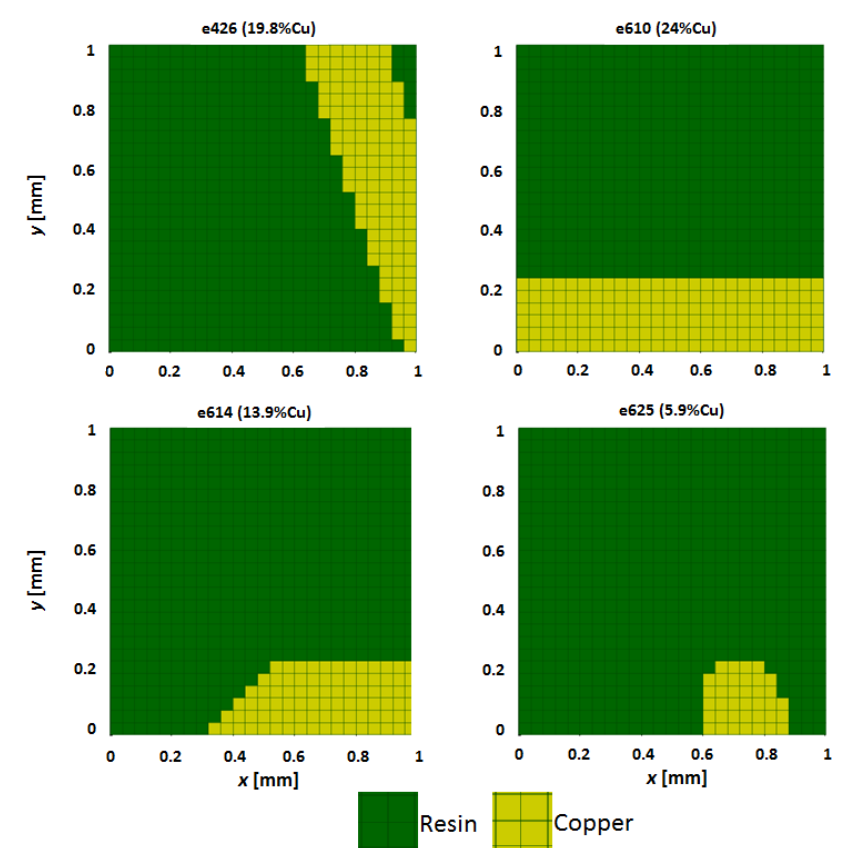

Figure 3 - Examples of real elements with the real copper and resin distribution. The area fractions of copper are of $19.8 \%$, $24 \%, 13.9 \%$ and $5.9 \%$, respectively. 


\subsection{Equivalence to a rectangle of copper}

The calculation of the equivalent rectangle is based on the moment of inertia tensor. The coordinates of copper's centre of mass are previously determined. The components of the inertia tensor are given by

$$
\mathbf{I}=\left[\begin{array}{ll}
I_{x x} & I_{x y} \\
I_{y x} & I_{y y}
\end{array}\right], \quad\left\{\begin{array}{l}
I_{x x}=\sum_{i=1}^{n} y_{i}{ }^{2} a_{i} \\
I_{y y}=\sum_{i=1}^{n} x_{i}{ }^{2} a_{i} \\
I_{x y}=-\sum_{i=1}^{n} x_{i} y_{i} a_{i} \\
I_{y x}=I_{x y}
\end{array}\right.
$$

$I_{x x}$ and $I_{y y}$ are the moments of inertia about the x- and y-axis, respectively. $I_{x y}$ and $I_{x y}$ have the same value and represent products of inertia. The distances from the copper's centre of mass to the centre of each point in the $x$ and $y$ axes are denoted as $x_{i}$ and $y_{i}$, respectively. The eigenvalues $\left(\lambda_{1}\right.$ and $\left.\lambda_{2}\right)$ are used to calculate the dimensions of a rectangle with an equivalent area of copper (see Figure 5) and the eigenvectors are used to determine its spatial orientation. For simplicity of calculation, the orientation of the equivalent rectangle is assumed to be 0 or 90 degrees according to the eigenvectors.

The area of the equivalent rectangle of copper is $b \times h$, with $b$ and $h$ given by,

$$
b=\sqrt[8]{144 \frac{\lambda_{1}{ }^{3}}{\lambda_{2}}}, \quad h=\sqrt[8]{144 \frac{\lambda_{2}{ }^{3}}{\lambda_{1}}}
$$
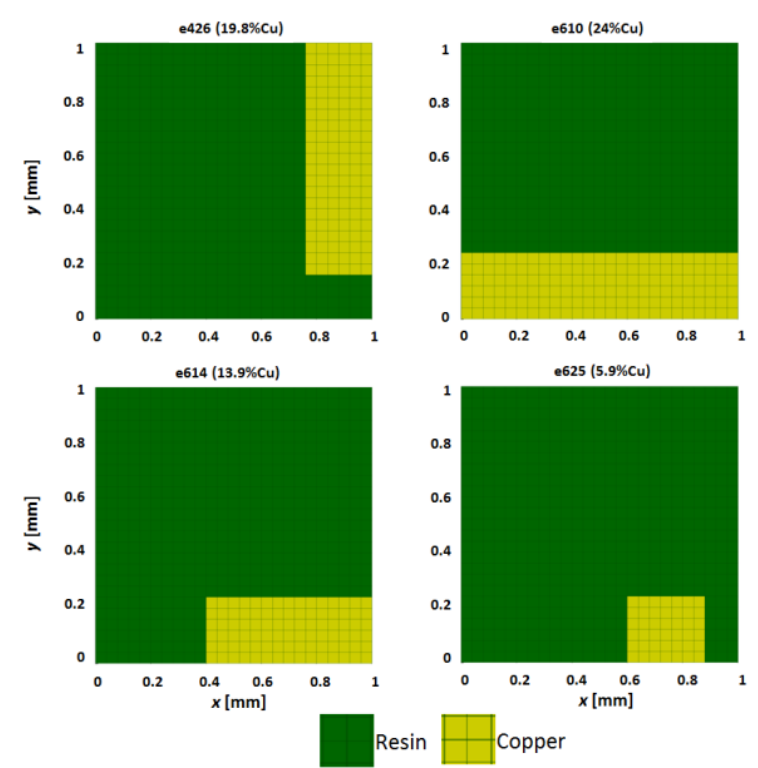

Figure 4 -Examples of the elements shown in Figure 3, in which the real copper distribution is replaced by an equivalent rectangle of copper determined to display the same inertia tensor and the same area fraction of copper. The area fractions of copper are of $19.8 \%, 24 \%, 13.9 \%$ and $5.9 \%$, respectively.
The centre of mass and the copper area fraction, $f_{c u}$, are the same as the original copper distribution. The sum of copper fraction and resin fraction, $f_{r e}$, is equal to 1 . The dimensions $b$ and $h$ are updated if the copper fraction of the equivalent rectangle if different from the copper fraction of the original configuration. The equivalent rectangles of the real element configurations (see Figure 3) are shown in Figure 4.

\subsection{Mathematical model}

The mathematical model for the determination of the homogenized Young modulus was based on the rule of mixtures. The rule of mixtures is based on the assumption of the equivalent stiffness, $R_{e q}$, which depends on the position of the bodies, i.e., if they are in parallel [eq. (5)] or in series [eq. (6)].

$$
\begin{gathered}
R_{e q}=R_{1}+R_{2} \\
R_{e q}=\left(\frac{1}{R_{1}}+\frac{1}{R_{2}}\right)^{-1}
\end{gathered}
$$

The relation between the stiffness, $R$, and the Young modulus, $E$, is expressed in eq. (7) and it depends on the area of the body's section, $A$, and the longitudinal length, $L$.

$$
R=\frac{E A}{L}
$$

For each direction, the element can be divided in 3 parts: one containing resin and copper ( $\alpha$-zone) and two others with resin only (see Figure 5). When tensioned, it is assumed that both materials of the $\alpha$-zone display the same displacement as they are tensioned in parallel.

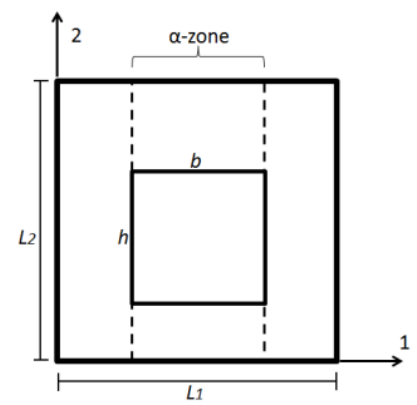

Figure 5 - Schema of the rule of mixtures for tension in direction 1 .

The total force is affected by the force applied to each material. For direction 1, the Young modulus of $\alpha$-zone $\left(E_{1 \alpha}\right)$ can be calculated with a simple relationship between the areas [see eq. (8)]. Along direction 1, the portions of resin and the $\alpha$-zone are placed in series and they are submitted to the same load. The element's total Young modulus along direction $1\left(E_{l}\right)$ is given by eq. (9).

$$
E_{1 \alpha}=\frac{L_{2}-h}{L_{2}} E_{r e s}+\frac{h}{L_{2}} E_{C u}
$$




$$
E_{1}=\left(\frac{b}{E_{1 \alpha} L_{1}}+\frac{\left(L_{1}-b\right)}{E_{r e s} L_{1}}\right)^{-1}
$$

\subsection{Comparing results}

The values of the Young modulus obtained with FEM and with the rule of mixtures are exposed in Figure 6. For each configuration, the Young modulus along $x$ and $y$ direction is analysed.

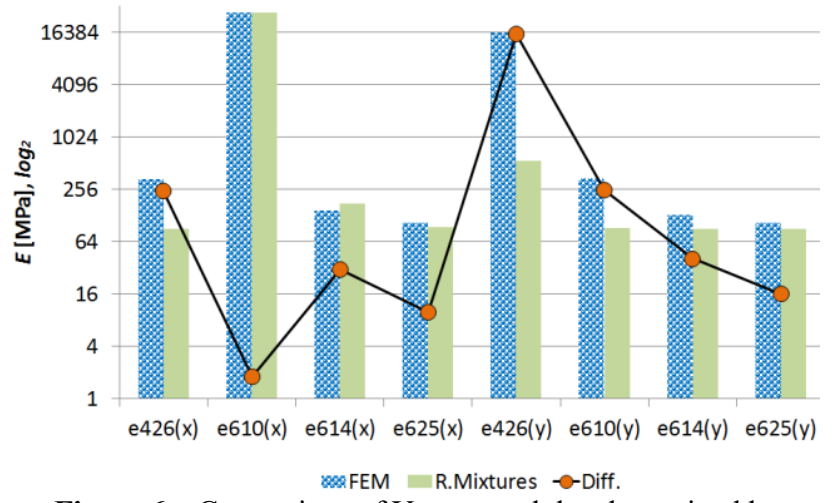

Figure 6 - Comparison of Young modulus determined by i) finite element computation and by using the

ii) homogenization technique based on the rule of mixtures.

The best result obtained is the Young modulus for element e610 in $x$ direction (Figure 6). On the other hand, the worst results were obtained for element e 426 in $x$ direction and for element e426 and e610 in $y$ direction. This is an indicator that the rule of mixtures provides good predictions when the distribution of copper is aligned with the tension test.

However, the copper can have infinite number of different distributions. And the homogenization method should provide better predictions in a much larger number of cases. Due to this reason, more complex copper distribution elements are used to improve a homogenization method in the next section.

\section{Homogenization procedure}

This homogenization method takes into account not only the calculation of equivalent properties but the characteristics of the elastic moduli matrix as well. In this section it is explained the homogenization method used to predict the Young modulus, Poisson ratio and thermal expansion properties of several multi-material elements of the copper layer of an arbitrary PCB.

This method has in consideration if the equivalent rectangle reaches opposite sides of the element for each direction. For each element it is calculated the dimensions of the equivalent rectangle by the same procedure as in the rule of mixtures. The complex copper distribution elements are shown in Figure 7 and its equivalent rectangles in Figure 8. The labels of these elements are e416, e424, e430 and e438.
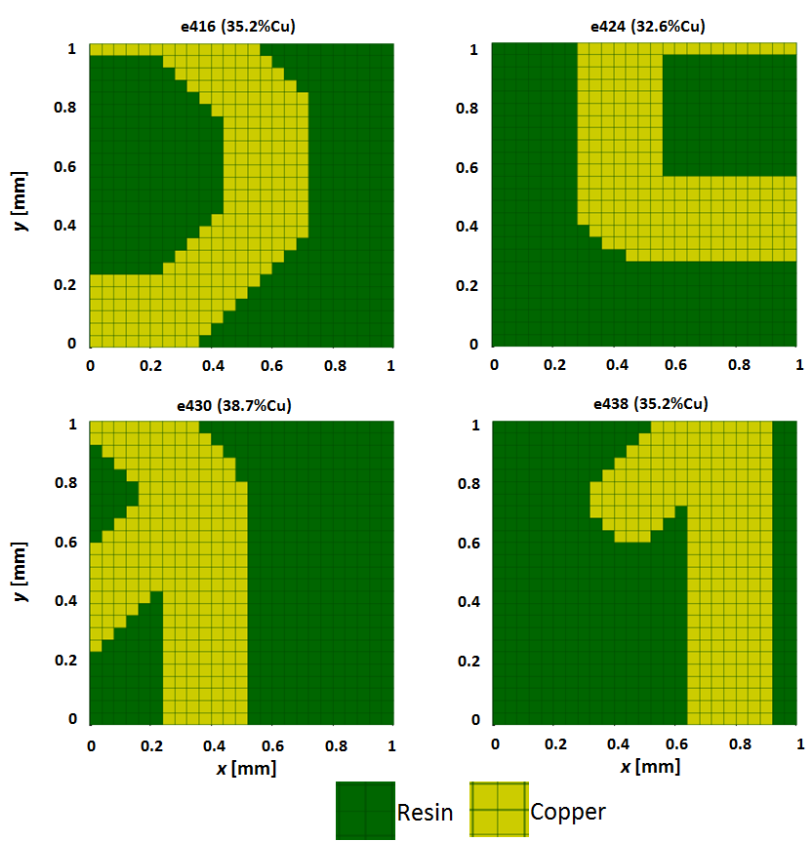

Figure 7 - Examples of real elements with a more complex geometrical distribution of copper and resin.
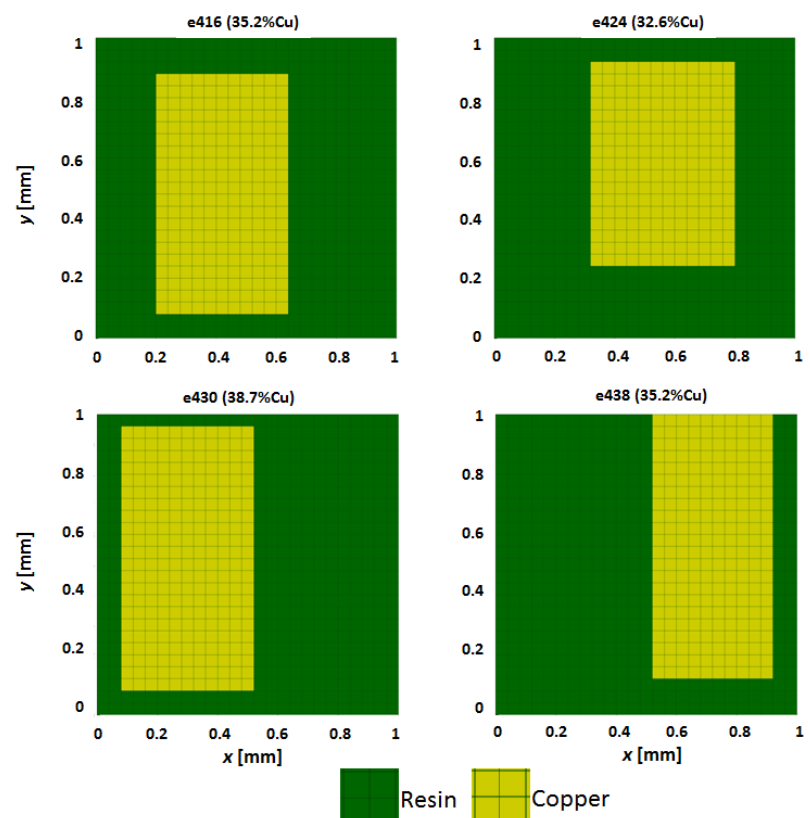

Figure 8 - Equivalent rectangles of the complex distributions of copper depicted in Figure 7.

In this part of the work it is studied the equivalent properties prediction of Young modulus, Poisson ratio and thermal expansion coefficient. However, it is explained only the procedure when the equivalent rectangle and the original copper distribution do not cross the element through opposite sides in any direction.

\subsection{Equivalent Young modulus}

The equivalent Young modulus prediction starts with the equivalent stiffness calculation through eq. (5) and (6). This previous equivalent stiffness, $R_{e q}$, is obtained for direction 1 and $2, R_{e q 1}$, and $R_{e q 2}$, respectively. For the direction 1, the procedure follows to eq. (10): 


$$
E_{1}=R_{M 1} \frac{L_{1}}{L_{2} L_{3}}
$$

where:

$$
R_{M 1}=R_{e q 1}\left(1+\frac{\left(E_{c u}\right)^{K_{0}}-\left(E_{r e}\right)^{K_{0}}}{\left(E_{r e}\right)^{K_{0}}}\right) C_{1}
$$

$K_{m}$ is obtained by eq. (12) and $C_{I}$ by eq. (13):

$$
\begin{gathered}
K_{m}=\left(\frac{E_{r e}}{1000}\right)^{k_{1}}\left(\frac{E_{c u}}{E_{r e}}\right)^{k_{2}}\left(\frac{v_{c u}}{v_{r e}}\right)^{k_{3}} \\
\left\{\begin{array}{l}
C_{1}=\frac{h_{r}}{15}\left(1+b_{r}-b_{r}^{3}\right)\left(3-b_{r}\right)\left(4+\frac{1}{h_{r}}\right) \\
b_{r}=b / L_{1} \\
h_{r}=h / L_{2}
\end{array}\right.
\end{gathered}
$$

The non-dimensional constants $k_{1}, k_{2}, k_{3}$ are set to $0.35,0.505$ and 3.8 , respectively, in order to get a better fitting to the FEM results. The constants were optimized according to an objective function that corresponds to the minimization of the differences between FEM and homogenization results. This homogenization method also checks if the original copper distribution crosses the element through opposite sides. This is done due to the good performance of the rule of mixtures in those cases, as it is possible to check the Young modulus ( $x$ direction) prediction in Figure 6. Due to this reason, the Young modulus in $z$ direction is calculated by the rule of mixtures as well.

\subsubsection{Comparing methods: simple elements}

The homogenized values of the Young modulus were obtained for each one of the simple elements (see Figure 3 ) and compared with the values from rule of mixtures, and summarized in Figure 9, with the values of the Young modulus in $x$ and $y$ directions are displayed as bars and the differences between the methods on the lines. The line of " $\Delta \mathrm{RM}$ " means the difference or error between FEM and rule of mixtures results. The line of " $\triangle$ Homog." indicates the error between FEM and homogenization method.

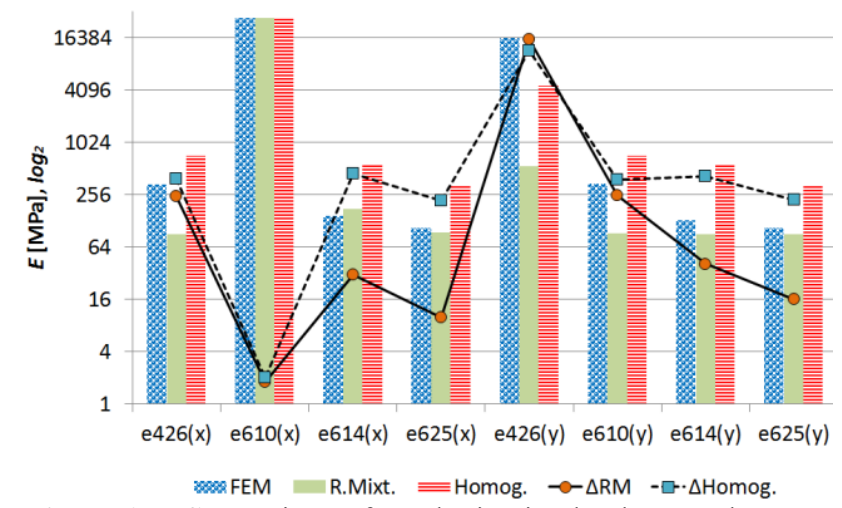

Figure 9 - Comparison of results in simple elements between the rule of mixtures and homogenization.
Most of the values of the rule of mixtures have lower differences than the homogenized ones. However, the rule of mixtures has the higher error: the $E_{y}$ of e 426 . The FEM value is 16206 [MPa], while the homogenization prediction is 4632 , but the rule of mixtures estimates only $553[\mathrm{MPa}]$. This element displays the largest error of the new homogenization method.

\subsubsection{Comparing methods: complex elements}

For each one of the complex elements (Figure 7), the homogenized values of the Young modulus are compared with the values from the rule of mixtures (Figure 6).

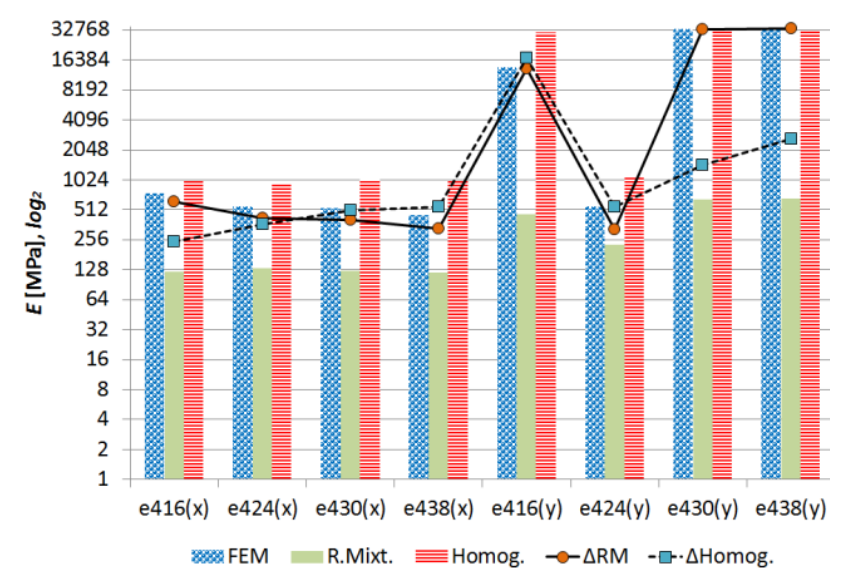

Figure 10 - Comparison of results of the complex elements between the rule of mixtures and the new homogenization technique.

Figure 10 indicates that the homogenization has a better performance than the rule of mixtures. The values of the rule of mixtures are always under $1 \mathrm{GPa}$ while some of the FEM values are over $10 \mathrm{GPa}$.

\subsection{Equivalent Poisson ratio}

In this section it is explained the homogenized Poisson ratio when a tensile deformation is applied along direction 1. Two values of Poisson ratio are calculated: $v_{12}$ and $v_{13}$ (eq. (14) and (15), respectively).

$v_{12}=\left(v_{c u}+C_{2}\right)\left[1-k_{4}\left(h_{r}-k_{5}\right)^{2}\right]$

$v_{13}=b_{r} v_{c u}\left(1+f_{r e} \frac{E_{r e}}{E_{c u}}\right) \frac{E_{1}}{E_{3}}$

where $C_{2}$ is obtained by the eq. (16),

$C_{2}=f_{r e}\left(\frac{v_{r e}-v_{c u}}{v_{c u}}\right)^{k_{6}}\left(\frac{E_{r e}-E_{c u}}{E_{c u}}\right)^{k_{7}} \sqrt{\frac{1-b_{r}}{K_{m}}}$

The non-dimensional constants $k_{4}, k_{5}, k_{6}$ and $k_{7}$ are set to $1.2,0.1,1.15$ and 4 , respectively. The constants were optimized for the minimization of the differences between FEM and homogenization results. This method 
was developed taking into consideration that the range of resin's Young modulus is lower than the range of copper's Young modulus. It was also assumed that the range of resin's Poisson ratio is higher than the range of copper's Poisson ratio.

Poisson ratio has a crucial impact on the mathematical calculation of FEM. The elastic moduli matrices have to be positive definite. That is taken into account in the equivalent Poisson ratio method: the error of Poisson ratio prediction by homogenization forces the elastic moduli matrix to be positive definite (check "4.1 Elastic moduli matrices"). Otherwise, no FEM simulation can be performed using homogenized properties.

The results for Poisson ratios $v_{x y}$ and $v_{y x}$ are shown in Figure 11 and Figure 12. The first four elements are the elements of Figure 3 and the last ones are the elements of Figure 7.

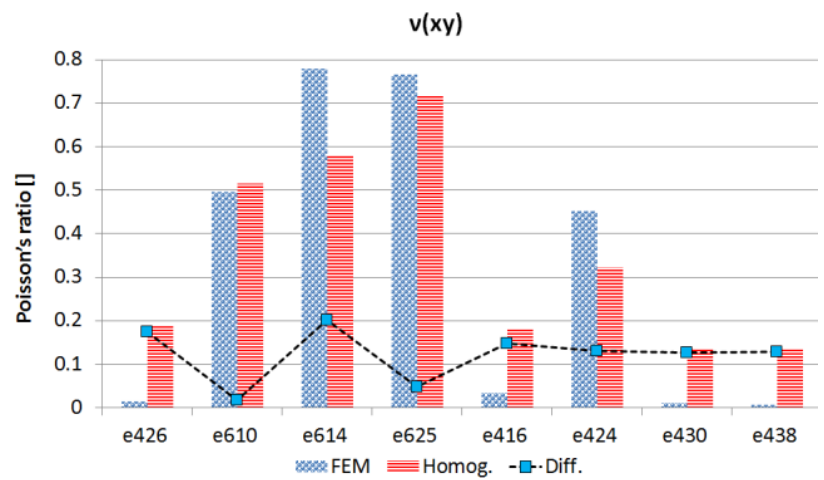

Figure 11 - Results of Poisson ratio $v_{x y}$.

These results show that the differences ("Diff." series) between homogenization and FEM method are mostly higher when Poisson ratio from FEM is lower than 0.2 and higher than 0.5 . It means that the homogenization method tends to provide a lower range of Poisson ratios. This is established in order to try to ensure that the elastic moduli are positive definite matrices.

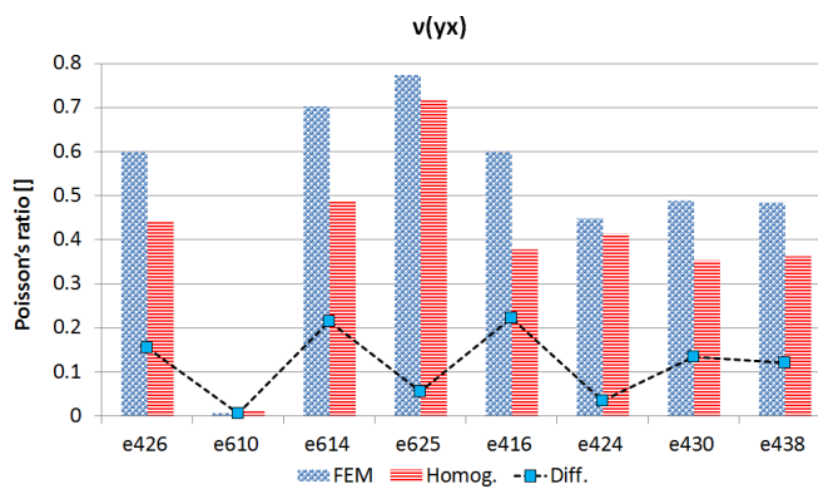

Figure 12 - Results of Poisson ratio $v_{y x}$

The results for Poisson ratios $v_{x z}$ and $v_{y z}$ are shown in Figure 13 and Figure 14, respectively. These values have special concerns because the copper crosses the element through opposite sides in $z$ direction.

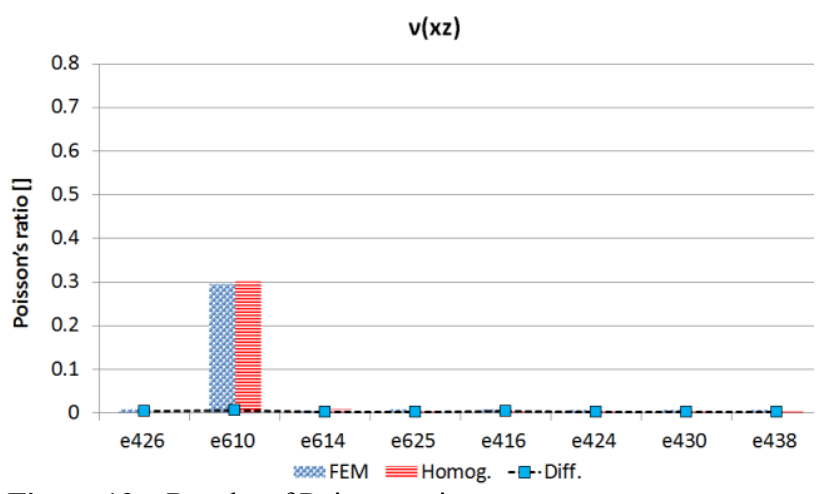

Figure 13 - Results of Poisson ratio $v_{x z}$.

When the extension is applied (in $x$ or $y$ direction), the contraction of the element is defined by the copper. The stiffness of copper is much higher than the resin stiffness. For this reason most of the Poisson ratios of Figure 13 and Figure 14 are nearly zero.

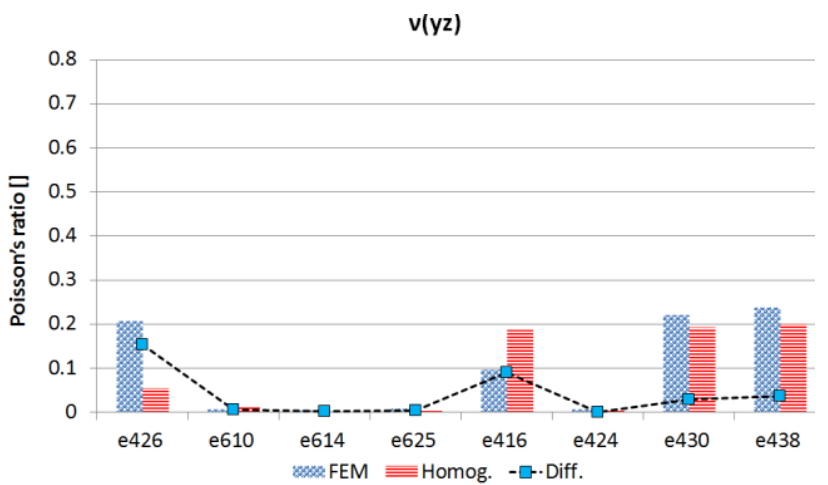

Figure 14 - Results of Poisson ratio $v_{y z}$.

However, the error of $v_{y z}$ in element e426 can be improved because the copper distribution crosses the element in $y$ direction. And that is not taken into account in this homogenization process.

\subsection{Equivalent thermal expansion}

The homogenized thermal expansion plays a paramount role on the analysis of warpage in PCBs. The calculation of the thermal expansion in direction $1, \alpha_{1}$, is based on eq. (17):

$$
\alpha_{1}=\frac{C_{3}\left(\alpha_{r e}+1\right)+b\left(\alpha_{c u}+1\right)-L_{1}}{L_{1}}
$$

where $C_{3}$ is obtained by the eq. (18):

$$
C_{3}=L_{1}-b+k_{8} \sqrt{L_{1}^{2}-b^{2}}+k_{9}\left(L_{1}-b\right)
$$

The dimensions of variables $\alpha_{1}, \alpha_{r e}$ and $\alpha_{c u}$, are $\left[\mathrm{m} \cdot \mathrm{m}^{-1} \cdot \mathrm{K}^{-1}\right]$. The non-dimensional constants $k_{8}$ and $k_{9}$ are set to 0.020 and 0.005 , respectively, in order to get a better matching between FEM results and the homogenization method. The error function corresponds to the minimization of the differences between FEM and homogenization results. 


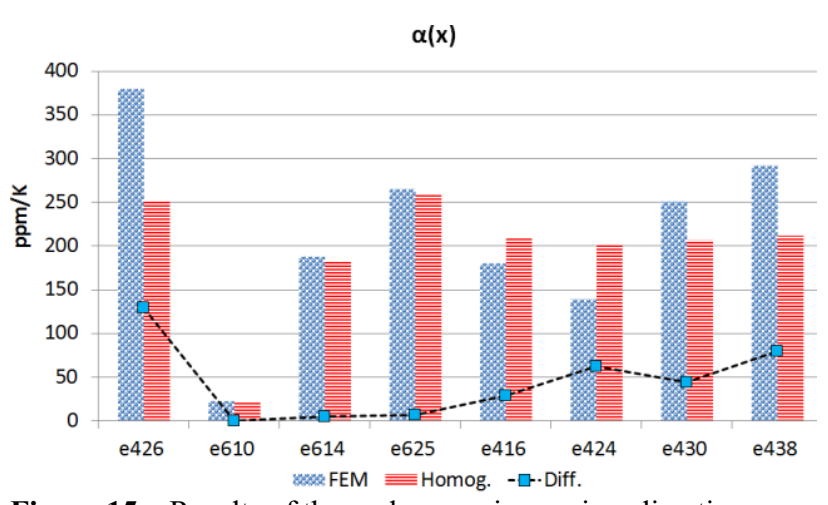

Figure 15 - Results of thermal expansion, $\alpha$, in $x$ direction.

The results of the thermal expansion in $\mathrm{x}$ and $\mathrm{y}$ direction is shown in Figure 15 and Figure 16, respectively.

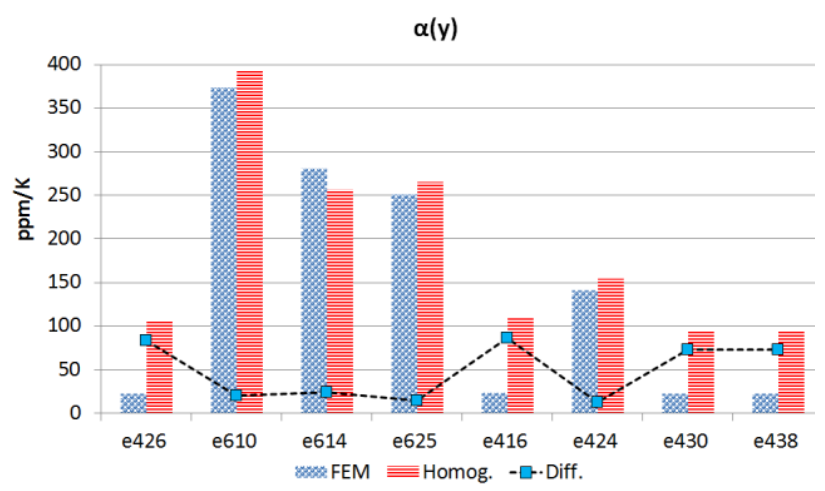

Figure 16 - Results of thermal expansion, $\alpha$, in $y$ direction.

The error of homogenization results is considerably high in some cases, e.g. see elements e426 and e438. A reason for that is the nonattendance to the original copper distribution. Once again, and similarly to the analysis of Figure 13 and Figure 14, the homogenization of the thermal expansion should take into consideration if the original copper distribution crosses the element at any direction.

\section{Global results analysis}

The homogenized values of the Young modulus, Poisson ratio and thermal expansion were obtained for each one of the 28 studied elements. In this section it is analysed the requirements of the elastic moduli, the time spent for each method calculation and the statistical analysis of all set of elements.

\subsection{Elastic moduli matrices}

The elastic moduli require special attention in a homogenization process. These matrices have to be positive definite. This aspect is hard to ensure during the homogenization process. When the elastic moduli is not a positive definite matrix, the global stiffness matrix of the FE problem becomes singular.

The worst results of homogenization are obtained with element e416 (see Figure 7). The first nine terms non-zero of the elastic moduli $(D)$ obtained by FEM and by homogenization are shown in eq. (19) and (20).

$$
\begin{aligned}
D_{F E M} & =\left[\begin{array}{ccc}
763 & 500 & 444 \\
504 & 14571 & 4513 \\
445 & 4511 & 44049
\end{array}\right] \\
D_{\text {hom }} & =\left[\begin{array}{ccc}
1073 & 450 & 252 \\
6362 & 35234 & 9818 \\
1763 & 9061 & 45273
\end{array}\right]
\end{aligned}
$$

The elastic moduli obtained by homogenization has all the diagonal terms higher than the moduli obtained by FEM. And some of the other terms are lower (see eq. (20)). However, matrix (20) is not a positive definite matrix given that the value of $D_{\text {hom }}(2,1)$ is too high. That is shown in eq. (21):

$$
\left(D_{\text {hom }(2,1)}\right)^{2}>\left(D_{\text {hom }(1,1)}\right)\left(D_{\text {hom }(2,2)}\right)
$$

Nevertheless, the elastic moduli has also to be a symmetric matrix. The one obtained by FEM is nearly symmetric. That is an indicator that the properties obtained by FEM may be more reliable. The elastic modulus $D_{\text {hom }}$ is forced to become symmetric, and thus symmetric terms are averaged. So, one obtains

$$
D_{\text {hom }}=\left[\begin{array}{ccc}
1073 & 3406 & 1008 \\
3406 & 35234 & 9439 \\
1008 & 9439 & 45273
\end{array}\right]
$$

After this process, the elastic moduli of element e416 is a positive definite matrix. It is interesting to take a look to the issue expressed in eq. (21). Due to inaccuracy of homogenization method, the value of $D_{\text {hom }}(2,1)$ was too high. However, the same accuracy of the method forced the values of $D_{\text {hom }}(1,1)$ and $D_{\text {hom }}(2,2)$ to be higher and the value of $D_{\text {hom }}(2,1)$ to be lower. So, after symmetrisation, the elastic moduli became a positive definite matrix.

\subsection{Time spent for each method}

The CPU time spent to calculate the elastic moduli is much lower through homogenization method than by FEM. The time spent by FEM is very dependent of the number of elements. In this analysis, the number of elements was $625(25 \times 25 \times 1)$ and the homogenization process was nearly 90 times faster than the FEM analysis.

\subsection{Statistical analysis}

The statistical analysis of the data obtained from the homogenization model and FEM can be performed using the coefficient of determination, $\mathrm{R}^{2}$, correlation and the analysis of the mean values and variance, as summarized in Table 2.

The requirements for $\mathrm{R}^{2}$ analysis are assumed. The Spearman's correlation indicates that the homogenization is a consistent model. However, the variances of the thermal expansion models are very different, i.e, higher than $20 \%$. 
Table 2 - Statistical data of the results

\begin{tabular}{|c|c|c|c|c|c|}
\hline & $\alpha(\mathrm{x})$ & $\alpha(\mathrm{y})$ & $E(\mathrm{x})$ & $E(\mathrm{y})$ \\
\hline \multicolumn{2}{|l|}{$\mathrm{R}^{2}$} & $76.5 \%$ & $92.9 \%$ & $99.6 \%$ & $94.6 \%$ \\
\hline \multicolumn{2}{|c|}{$\begin{array}{l}\text { Spearman's } \\
\text { correlation }\end{array}$} & 0.896 & 0.894 & 0.981 & 0.984 \\
\hline \multirow{2}{*}{ Mean } & FEM & 181 & 194 & 5.12 & 11.73 \\
\hline & Homo. & 179 & 217 & 5.39 & 12.08 \\
\hline \multirow{2}{*}{ Variance } & FEM & 9794 & 20284 & 98.58 & 323.33 \\
\hline & Homo. & 6799 & 13998 & 97.50 & 322.20 \\
\hline
\end{tabular}

When the differences between models of thermal expansion ( $x$ direction) and Young modulus ( $y$ direction), several outliers are found in the respective boxplot (Figure 17). This is an indicator that the homogenization method still needs to be improved because it clearly fails in some very particular cases: when the elastic property is under $1 \mathrm{GPa}$ and when the copper distribution crosses the element in $x$ or $y$ direction.
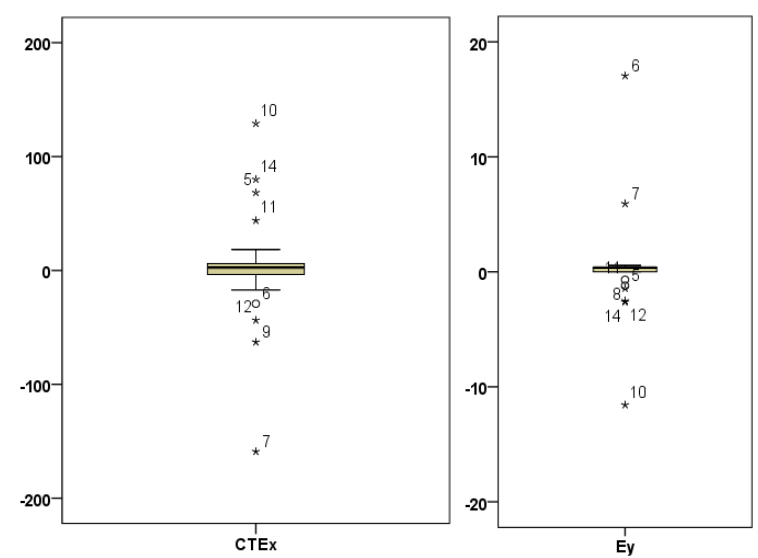

Figure 17 - Boxplot of the differences between FEM and homogenization model for $\alpha_{x}$ (left) and $E_{y}$ (right).

The number of outliers in the boxplots of Figure 17 also indicates that the sample should be bigger and the elements should represent a larger range of copper distributions.

\section{Conclusions}

The homogenization method is a reliable alternative to the FEM simulation for equivalent properties calculation. However, there are opportunities to develop the method regarding less accurate aspects.

Concerning Young modulus calculation, there is a lack of accuracy in the homogenised properties when the elastic property is under $1 \mathrm{GPa}$. Maybe $k_{l}$ optimization should be improved in eq. (12). A similar observation can be made for Poisson ratio. When the Poisson ratio from FEM is lower than 0.2 and higher than 0.5 , the homogenised property loses accuracy.

The error of Poisson ratio and thermal expansion can be reduced because in some elements (e.g. element e416 and e430) the copper distribution crosses the element in $y$ direction. The homogenization process does not have this aspect in consideration. By this way, the accuracy of the model will be improved for some copper distributions (e.g. for element e426).
The homogenization method provides an effective way of properties prediction. In general, the values obtained through this method are acceptable for warpage prediction because $\mathrm{R}^{2}$ and Spearman's correlation are higher than 0.75 and 0.8 , respectively. Moreover, the homogenization method is much faster (90 times faster) than FEM method and provides positive definite matrices of the elastic moduli.

Finally, other copper distributions should be used to test the homogenization model in order to get a better analysis of the range and the potential of this method.

\section{Acknowledgements}

This research is sponsored:

- by the Portugal Incentive System for Research and Technological Development. Project in co-promotion $n^{\circ}$ 36265/2013 (Project HMIExcel - 2013-2015), and - by FCT with the reference project UID/EEA/04436/2013, by FEDER funds through the COMPETE 2020 - Programa Operacional Competitividade e Internacionalização (POCI) with the reference project POCI-01-0145-FEDER-006941.

\section{References}

1. S.T. Pantelides, et al., Performance, reliability, radiation effects, and aging issues in microelectronics - From atomic-scale physics to engineering-level modeling 54, 9 (2010)

2. F. Clyde and J. Coombs, Printed Circuits Handbook. (2008): McGraw-Hill.

3. P. Hutapea and J. Grenestedt, Effect of temperature on elastic properties of woven-glass epoxy composites for printed circuit board applications 32, (2003)

4. L. McCaslin and K. Sitaraman. Methodology for modeling substrate warpage using copper trace pattern implementation. in Electronic Components and Technology Conference. (2008).

5. S.-W. Kim, et al., Simulation of warpage during fabrication of printed circuit boards 1, (2011)

6. S. Timoshenko, Theory of Elasticity. (1951): McGraw-Hill.

7. A.K. Kaw, Mechanics of Composite Materials. (2006): CRC Press.

8. P. Hutapea and J. Grenestedt, Tuning of electric artworks of printed circuit boards to reduce warpage (2004) 